\title{
Macroscopic Satellite Nodule Not Identified
}

National Cancer Institute

\section{Source}

National Cancer Institute. Macroscopic Satellite Nodule Not Identified. NCI Thesaurus.

Code C160828.

The macroscopic satellite nodule is not identified. 